\title{
Acoustoelectric effects in very high-mobility $p$-SiGe/Ge/SiGe heterostructure at low temperatures in high magnetic fields
}

\author{
I.L. Drichko, ${ }^{1}$ V.A. Malysh, ${ }^{1}$ I.Yu. Smirnov, ${ }^{1}$ A.V. Suslov, ${ }^{2}$ O.A. Mironov, ${ }^{3}$ M. Kummer, ${ }^{4}$ and H. von Känel ${ }^{4}$ \\ ${ }^{1}$ A.F. Ioffe Physical-Technical Institute of the Russian Academy of Sciences, 194021 St.Petersburg, Russia \\ ${ }^{2}$ National High Magnetic Field Laboratory, Tallahassee, FL 32310, USA \\ ${ }^{3}$ Warwick SEMINANO R $\xi D$ Centre, University of Warwick Science Park, Coventry CV4 7EZ, UK \\ ${ }^{4}$ Laboratorium für Festkörperphysik ETH Zürich, CH-8093 Zürich Switzerland
}

(Dated: August 22, 2018)

\begin{abstract}
The contactless Surface Acoustic Wave (SAW) technique was implemented to probe the highfrequency (ac) conductivity in a high-mobility $p$-SiGe/Ge/SiGe structure in the integer quantum Hall (IQHE) regime. The structure was grown by low-energy plasma-enhanced chemical vapor deposition and comprised a two-dimensional channel formed in a compressively strained Ge layer. It was investigated at temperatures of $0.3-5.8 \mathrm{~K}$ and magnetic fields up to $18 \mathrm{~T}$ at various SAW intensities. In the IQHE regime, in minima of the conductivity oscillations with small filling factors, holes are localized. The ac conductivity is of the hopping nature and can be described within the "two-site" model. Furthermore, the dependence of the ac conductivity on the electric field of the SAW was determined. The manifestation of non-linear effects is interpreted in terms of nonlinear percolation-based conductivity.
\end{abstract}

PACS numbers: $73.63 . \mathrm{Hs}, 73.50 . \mathrm{Rb}$

\section{INTRODUCTION}

Earlier in Ref. 1 we applied the acoustic method for the investigation of a high-mobility $p$-SiGe/Ge/SiGe structure with a hole concentration of $\mathrm{p}=6 \times 10^{11} \mathrm{~cm}^{-2}$ grown by low-energy plasma-enhanced chemical vapor deposition (LEPECVD) $2 \underline{2}$ The measurements were performed as a function of temperature $(1.6-4.2 \mathrm{~K})$ and the magnetic field (up to $8.4 \mathrm{~T}$ ). We determined the general conduction parameters of a two-dimensional hole gas in the linear regime: hole density and mobility, effective mass, quantum and transport relaxation times, as well as the Dingle temperature. In the non-linear regime we extracted the values of the energy relaxation time and deformation potential. In our previous work, we found good agreement between the data obtained from the contactless acoustic technique and those from dc measurements, published in Ref. 3 .

The quantum Hall effect in multilayer $p$-Ge/GeSi heterostructures, grown by the hydride method (chemical vapor deposition), was first observed in the early 90's by the authors of Ref. 4. Studies of the magnetoresistance, carried out in a tilted magnetic field in Ref. 5, have shown that the SdH oscillation pattern of the spin subsystem substantially depends on the degree of strain in the conducting layers. In the case of a high compressive strain the oscillation pattern is completely determined by the magnetic field component normal to the sample surface, 6.7 These authors also pointed out the work of Ref. 8, in which the hole energy spectrum in the first size-quantized subband was predicted to be significantly non-parabolic, resulting in the dependence of the effective mass and the g-factor on the Fermi energy, i.e., on the hole concentration.

The authors of Ref. 9 studied samples with a single $p$-Ge/GeSi quantum well, grown by molecular beam epi- taxy, and having hole concentration in the wide range of $(0.57-2.1) \times 10^{12} \mathrm{~cm}^{-2}$. They indeed observed a density dependent effective mass. A marked dependence of the effective mass on the concentration was also observed in Ref. 10 in single $p$-Ge/GeSi quantum wells with high mobility grown by LEPECVD. These works confirmed experimentally the prediction of non-parabolicity of the heavy hole band in strained Ge quantum wells.

The properties of $p$-Ge/GeSi structures were previously probed mainly by direct current transport measurements of the magnetoresistance or cyclotron resonance experiments. Therefore, it was useful to investigate those structures with contactless acoustic methods allowing calculations of the high-frequency conductivity. 1

As the study in Ref. 1 was mainly focused on the properties of a delocalized two-dimensional hole gas (2DHG), it seemed logical to extend those measurements to identify the low-temperature conduction mechanisms in the hole localization regime. Moreover, the value of the gfactor is of a great interest in these structures. In fact, the determination of this value turned out to be complicated in Ref. 1, as the spin splitting (at odd filling numbers $\nu=5$ and 7 ) was barely resolved even at $T=$ $1.6 \mathrm{~K}$. Thus, the present work also addresses the problem of determining the g-factor. For studying holes in the localized regime and determining the g-factor, it was necessary to carry out the measurements at lower temperatures and at higher magnetic fields to observe oscillations corresponding to the spin-split Landau levels with smaller filling factors and greater oscillation amplitude. 


\section{EXPERIMENTAL RESULTS}

\section{A. Object}

Here we studied the $p$-SiGe/Ge/SiGe sample (K6016) the conductivity of which was investigated earlier by direct current $^{3}$ and $\mathrm{SAW}: \underline{1}$ The layer structure of the sample is illustrated in Fig. 1(a).

In this system the 2D channel is formed in a compressively strained modulation doped Ge layer. Due to the strain the threefold degenerated valence band of $\mathrm{Ge}$ is split into 3 subbands, the highest of which is occupied by heavy holes.

The entire structure was grown by LEPECVD, by making use of the large dynamic range of growth rates offered by that technique..$^{\frac{3}{}}$ The buffer, graded at a rate of about $10 \% / \mu \mathrm{m}$ to a final Ge content of $70 \%$, and the $4 \mu \mathrm{m}$ thick constant composition layer were grown at a high rate of $5-10 \mathrm{~nm} / \mathrm{s}$ while gradually lowering the substrate temperature $T_{s}$ from $720^{\circ} \mathrm{C}$ to $450^{\circ} \mathrm{C}$. The active layer structure, consisting of a pure $20 \mathrm{~nm}$ thick Ge layer sandwiched between cladding layers with a Ge content of about $60 \%$ and a $\mathrm{Si}$ cap, was grown at a low rate of about $0.3 \mathrm{~nm} / \mathrm{s}$ at $T_{s}=450^{\circ} \mathrm{C}$. Modulation doping was achieved by introducing dilute diborane pulses into the cladding layer grown above the channel after an undoped spacer layer of about $30 \mathrm{~nm}$.

\section{B. Method}

The experimental setup is shown in Fig. 1(b). A surface acoustic wave (SAW) is excited on one side of a piezoelectric platelet $\left(\mathrm{LiNbO}_{3}\right)$ by an inter-digital transducer. The SAW propagating along the plane of the lithium niobate is accompanied by a high-frequency electric field. This electric field penetrates into the 2D channel located in the semiconductor structure which is slightly pressed onto the surface of the platelet by means of springs. The field produces electrical currents which, in turn, cause Joule losses. As a result of the interaction of the SAW electric field with charge carriers in the quantum well, the SAW attenuation and its velocity change are governed by the complex high-frequency conductivity, $\sigma^{a c}(\omega)$.

This "sandwich-like" experimental configuration enables contactless acoustoelectric experiments on nonpiezoelectric 2D systems, such as $\mathrm{SiGe} / \mathrm{Ge} / \mathrm{SiGe}$. The technique was first employed in Ref. 11 for $\mathrm{GaAs} / \mathrm{Al}_{x} \mathrm{Ga}_{1-x} \mathrm{As}$ heterostructures.

\section{Experiment \\ 1. Linear regime}

The measurements of the SAW attenuation $\Delta \Gamma=$ $\Gamma(B)-\Gamma(B=0)$ and velocity change $\Delta v / v$ in the $p$ -

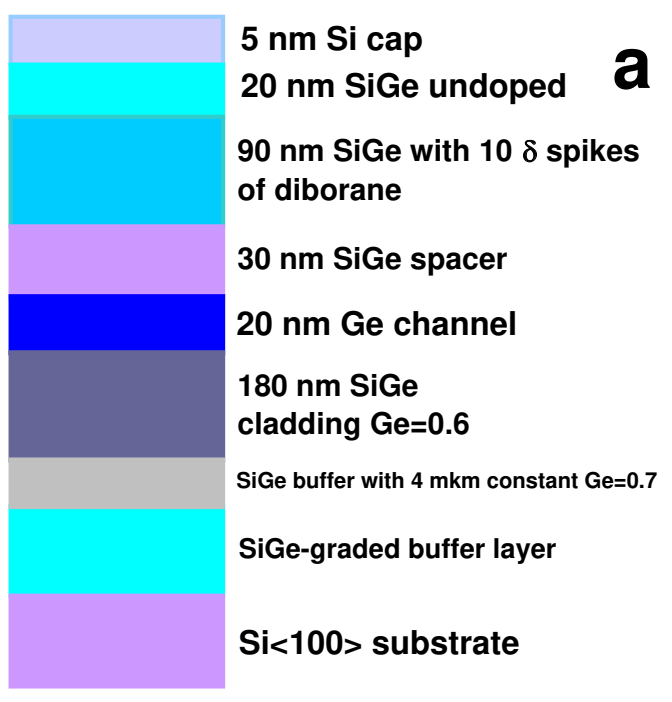

$5 \mathrm{~nm}$ Si cap

$20 \mathrm{~nm}$ SiGe undoped

$90 \mathrm{~nm}$ SiGe with $10 \delta$ spikes

of diborane

$30 \mathrm{~nm}$ SiGe spacer

$20 \mathrm{~nm}$ Ge channel

$180 \mathrm{~nm} \mathrm{SiGe}$

cladding $\mathrm{Ge}=0.6$

SiGe buffer with $4 \mathrm{mkm}$ constant $\mathrm{Ge}=0.7$

SiGe-graded buffer layer

$\mathrm{Si}<100>$ substrate
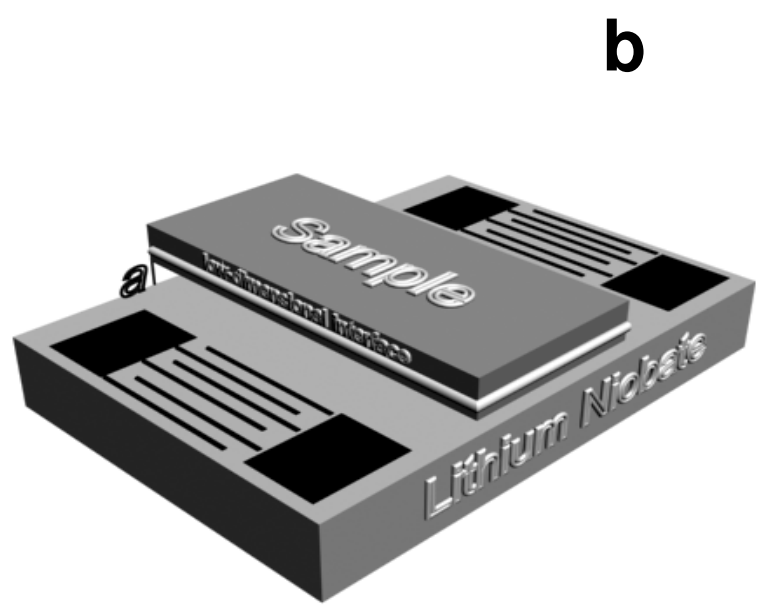

FIG. 1: (a) Cross-section of the studied sample and (b) sketch of the acoustic experiment setup.

GeSi/Ge/GeSi heterostructure, containing a single quantum well with a hole density of $\mathrm{p}=6 \times 10^{11} \mathrm{~cm}^{-2}$, were done at frequencies of 30 and $85 \mathrm{MHz}$ in magnetic fields $B$ up to $18 \mathrm{~T}$ and in the temperature range of $0.3-5.8$ K. The top panel of Fig. 2a shows the magnetic field dependence of the attenuation $\Gamma(B)$, obtained at a temperature of $0.3 \mathrm{~K} . \Gamma(B)$ is equal to the experimentally measured $\Delta \Gamma(B)$, since $\Gamma(B=0) \ll \Gamma(B)$ for a zerofield conductivity $\sigma(B=0) \approx 6 \times 10^{-3} \Omega^{-1}$. The bottom panel of Fig. 2 illustrates the SAW velocity change $\Delta v(B) / v(0) \equiv[v(B)-v(0)] / v(0)$ under the same conditions. Analogous curves were obtained for the other temperatures. Fig. 2 $\mathrm{b}$ shows the low field portion of the same curves corresponding to the magnetic field region from 0 to $5.5 \mathrm{~T}$.

One can see that both the absorption coefficient and the velocity change undergo Shubnikov-de Haas ( $\mathrm{SdH}$ ) type oscillations in the magnetic field. The rich oscillation pattern appears at filling factor as large as $\nu=22$.

From the measured values of $\Gamma$ and $\Delta v / v$ both the real part $\sigma_{1}$ and imaginary part $\sigma_{2}$ of the high-frequency (ac) 

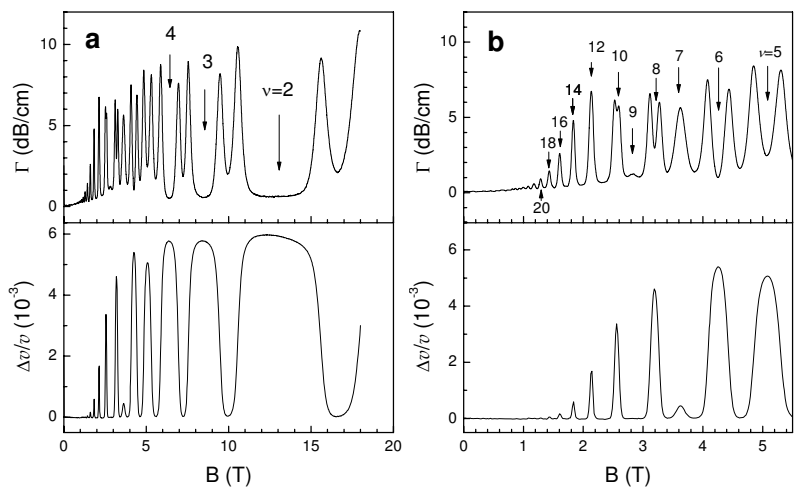

FIG. 2: a) Magnetic field dependences of $\Gamma$ and $\Delta v / v(0)$ up to $18 \mathrm{~T}$; b) $\Gamma$ and $\Delta v / v(0)$ in fields of up to $5.5 \mathrm{~T}$. Arrows denote the filling factors. $f=30 \mathrm{MHz} ; T=0.3 \mathrm{~K}$.

conductivity $\sigma^{\text {ac }}(\omega) \equiv \sigma_{1}-i \sigma_{2}$ could be calculated using the following equations taken from Ref. 12:

$$
\begin{aligned}
& \Gamma=8.68 \frac{K^{2}}{2} q A \frac{4 \pi \sigma_{1} t(q) / \varepsilon_{s} v}{\left[1+4 \pi \sigma_{2} t(q) / \varepsilon_{s} v\right]^{2}+\left[4 \pi \sigma_{1} t(q) / \varepsilon_{s} v\right]^{2}} \\
& \text { where } A=8 b(q)\left(\varepsilon_{1}+\varepsilon_{0}\right) \varepsilon_{0}^{2} \varepsilon_{s} \exp [-2 q(a+d)], \text { and } \\
& \frac{\Delta v}{v}=\frac{K^{2}}{2} A \frac{1+4 \pi \sigma_{2} t(q) / \varepsilon_{s} v}{\left[1+4 \pi \sigma_{2} t(q) / \varepsilon_{s} v\right]^{2}+\left[4 \pi \sigma_{1} t(q) / \varepsilon_{s} v\right]^{2}},
\end{aligned}
$$

where $K^{2}$ is the electro-mechanic coupling constant for lithium niobate (in our case, the $\mathrm{Y}$-cut), $q$ and $v$ are the SAW wave vector and velocity in $\mathrm{LiNbO}_{3}$, respectively; $a$ is the gap between the piezoelectric platelet and the sample, $d$ is the distance between the sample surface and the $2 \mathrm{D}$ channel, which is determined as described in Ref. 12; $\varepsilon_{1}, \varepsilon_{0}$ and $\varepsilon_{s}$ are the dielectric constants of $\mathrm{LiNbO}_{3}$, of vacuum, and of the semiconductor, respectively; $b$ and $t$ are functions of $a, d, \varepsilon_{1}, \varepsilon_{0}$ and $\varepsilon_{s}$.

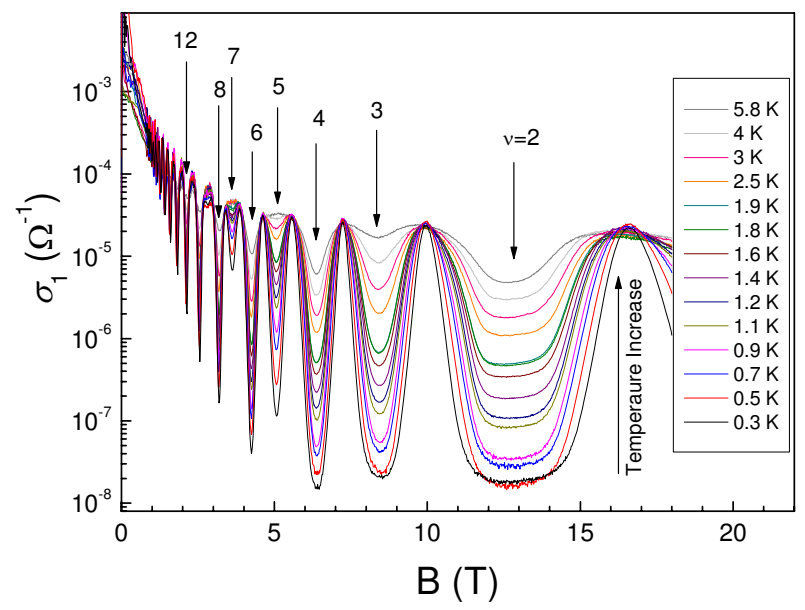

FIG. 3: Dependence of $\sigma_{1}$ at $30 \mathrm{MHz}$ on a magnetic field at different temperatures. The filling factors are marked by arrows.

The magnetic field dependence of $\sigma_{1}$, i.e., the real part of the complex high-frequency conductivity, obtained from the SAW measurements at different temperatures is shown in Fig. 3.

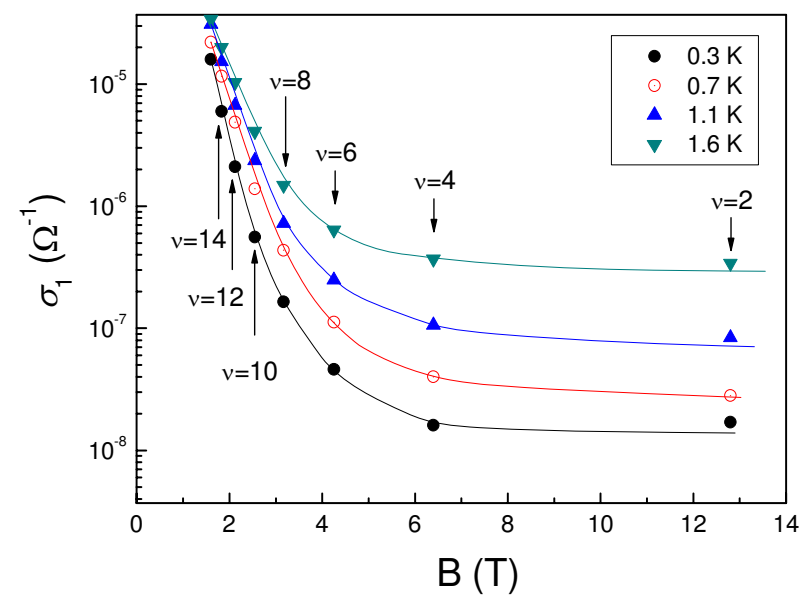

FIG. 4: Magnetic field dependence of $\sigma_{1}$ in minima of the $\mathrm{dB}$ oscillations with filling factors $\nu=2,4,6,8,10,12$ and 14 at $\mathrm{cm}$ temperatures $0.3,0.7,1.1$ and $1.6 \mathrm{~K}$.

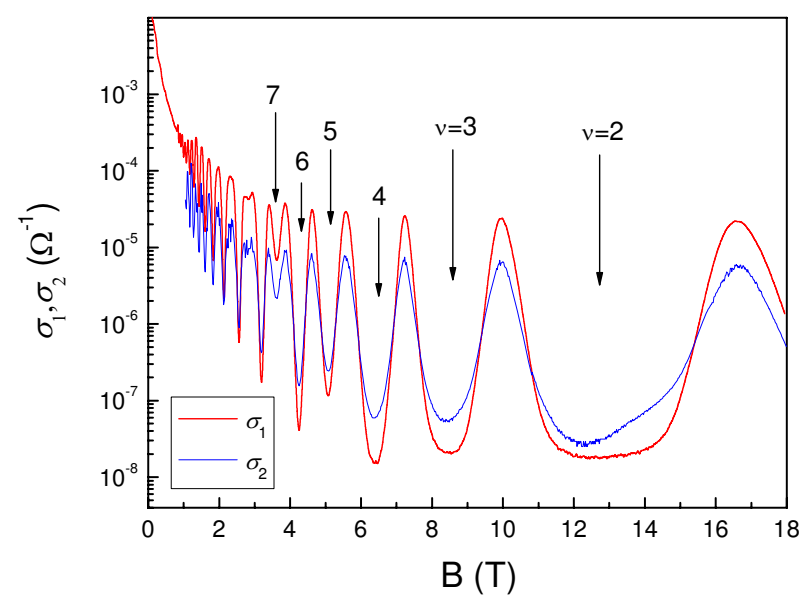

FIG. 5: Magnetic field dependences of the real $\sigma_{1}$ and imaginary $\sigma_{2}$ parts of the ac conductivity; $T=0.3 \mathrm{~K}, f=30 \mathrm{MHz}$.

The real part of the ac conductivity in the minima of oscillations with even filling factors at different temperatures is presented in Fig. 4. For magnetic fields in the range of $1 \mathrm{~T}<B<6 \mathrm{~T}$ we find that $\sigma_{1} \propto 1 / B^{2}$, while above $B>6 \mathrm{~T} \sigma_{1}$ is virtually independent of the magnetic field.

The real $\sigma_{1}$ and imaginary $\sigma_{2}$ parts of the ac conductivity in magnetic fields up to $18 \mathrm{~T}$ and at a temperature $T=0.3 \mathrm{~K}$ are displayed in Fig. [5. It can be seen that in strong fields, corresponding to small filling factors, $\sigma_{1}<\sigma_{2}$ in the minima of the oscillations. Yet, on the contrary, $\sigma_{1}$ in its oscillation maxima tends to be greater than $\sigma_{2}$. At larger filling factors the minimum of $\sigma_{2}$ at first equals that of $\sigma_{1}($ at $\nu=10)$ and then $\sigma_{2}$ becomes far smaller than $\sigma_{1}$ for $\nu \geq 12$. 

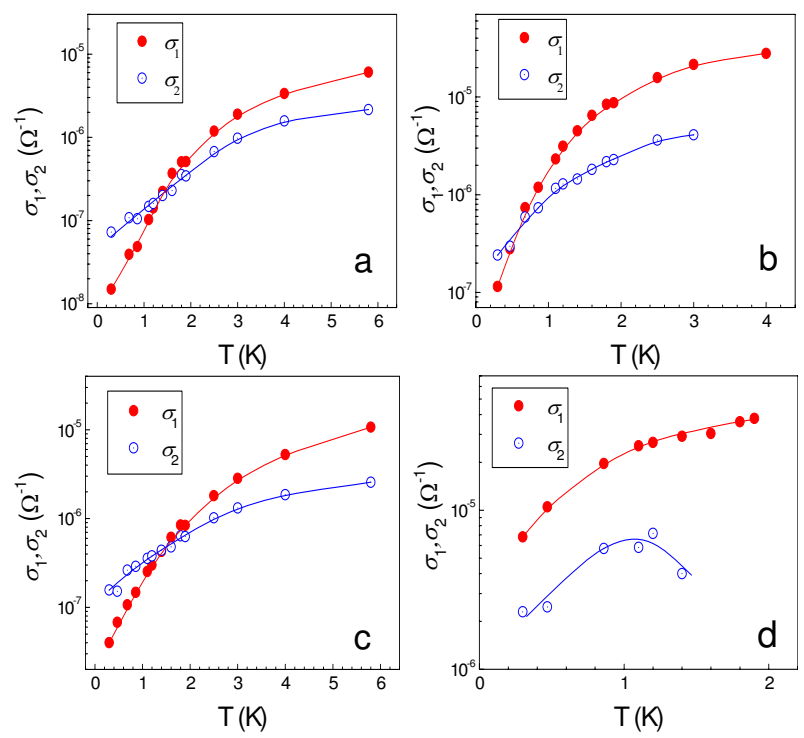

FIG. 6: Temperature dependence of the real $\sigma_{1}$ and imaginary $\sigma_{2}$ parts of the ac conductivity for different filling factors: a) $\nu=4$, b) $\nu=5$, c) $\nu=6$, d) $\nu=7$. The lines are guides to the eye.

Figure 6 shows how the conductivities $\sigma_{1}$ and $\sigma_{2}$ in the minima of oscillations with different filling factors vary with the temperature.

It is seen that $\sigma_{2}>\sigma_{1}$ for small filling factors at low temperatures. With increasing temperature the relation between the complex conductivity components changes, and $\sigma_{1}$ becomes greater than $\sigma_{2}$. For filling factor $\nu=7$ $\sigma_{2}<\sigma_{1}$ in the whole temperature range.

The whole set of experimental dependences of the ac conductivity on magnetic field and temperature in the oscillation minima at high magnetic fields and low temperatures as well as the condition $\sigma_{2}>\sigma_{1}$ indicates a hopping nature of the high-frequency conductivity, which can be described within a "two-site" model 13,14

In relatively small magnetic fields $(B<2 \mathrm{~T})$ holes are delocalized, and $\sigma_{2} \ll \sigma_{1}$. In this case the analysis of the temperature damping of the $\mathrm{SdH}$ oscillations allows the carrier parameters such as effective mass, Dingle temperature, and quantum relaxation time to be evaluated. These values are close to the ones which we determined for this sample in Ref. 1

\section{Determination of the g-factor}

To obtain the g-factor value we analyzed the temperature dependence of the conductivity at odd filling factors $\nu=3,5$, and 7 . In the temperature range where the Arrhenius law is valid, the real part of the ac conductivity is described by the equation: $\sigma_{1} \propto \exp \left(-\Delta E / 2 k_{B} T\right)$ (where $\Delta E=g \mu_{B} B, g$ is the $g$-factor, $\mu_{B}$ is the Bohr magneton. Thus, the slope of the linear dependence in the Arrhenius plot allows the activation energy $\Delta E$ to be determined for each $\nu$ (Fig. (7). The slope of the depen- dences $\Delta E(B)$, in turn, yields the value of the g-factor. We find $g=4.5 \pm 0.3$ (see inset in Fig. 7).

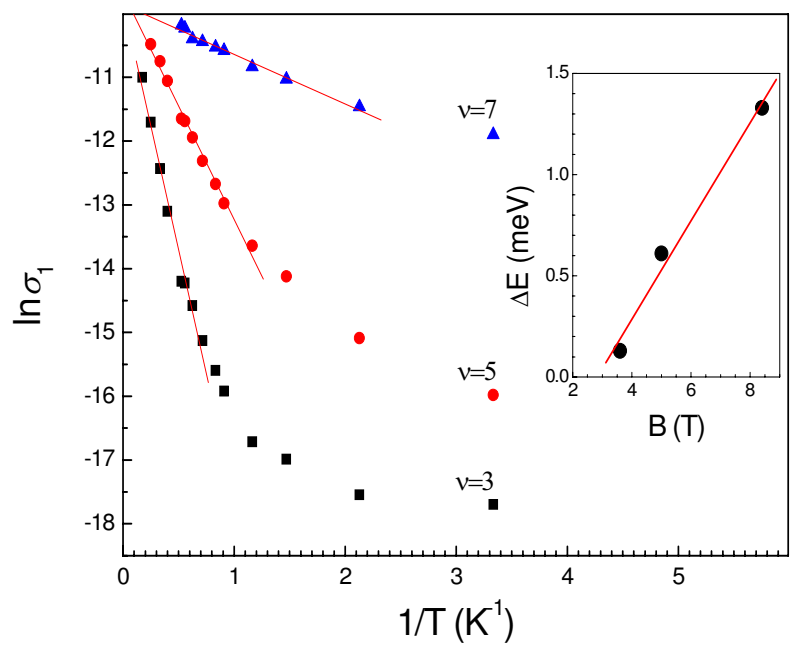

FIG. 7: Arrhenius plots of $\sigma_{1}$ at various odd filling factors. Inset: The activation energy in magnetic field for odd filling factors. Lines are results of the linear fitting.

Figure 17 demonstrates that the $\ln \sigma_{1}$ exhibits a linear dependence on the inverse temperature, corresponding to an Arrhenius law, down to temperatures of about $T \approx(0.5 \div 1) \mathrm{K}$. At lower temperatures the conductivity has a weaker temperature dependence, which is usually associated with a transition to hopping conductivity.

In the structures under study the two-dimensional channel is located in a compressively strained Ge quantum well. It is known that in two dimensional Ge and Si layers the compressive strain lifts the degeneracy of the heavy and light holes of the valence band pushing up the heavy hole energy subband. As stated in Ref. 8, the hole bands in the two-dimensional objects with a structurally complicated valence band, such as thin Ge layers, are non-parabolic. The value of the g-factor, therefore, depends on the Fermi energy. Indeed, at the bottom of the heavy hole band $g=20.4, \frac{15}{,}$ while in the system with multiple quantum wells $p$-Ge/GeSi with density $\mathrm{p} \approx 1 \times 10^{11} \mathrm{~cm}^{-2} \mathrm{~g}=14 \pm 1.4, \frac{15}{\text { to }}$ to be compared with our present result of $g=4.5 \pm 0.3$ for $\mathrm{p}=6 \times 10^{11} \mathrm{~cm}^{-2}$. This is consistent with the findings of Ref. 8 .

\section{Nonlinear regime}

The ac conductivity response to the larger power of the surface acoustic waves, i.e., the response in the non-linear regime, was also examined in this work.

The absorption coefficient $\Gamma$ and velocity change $\Delta v / v$ in magnetic fields up to $18 \mathrm{~T}$ are displayed in Figure 8 at different SAW powers.

Figure 9 shows the magnetic field dependence of $\sigma_{1}$ at different SAW powers, calculated using equations 1 and 2 . Here it is seen that with rising power the ac conductivity 


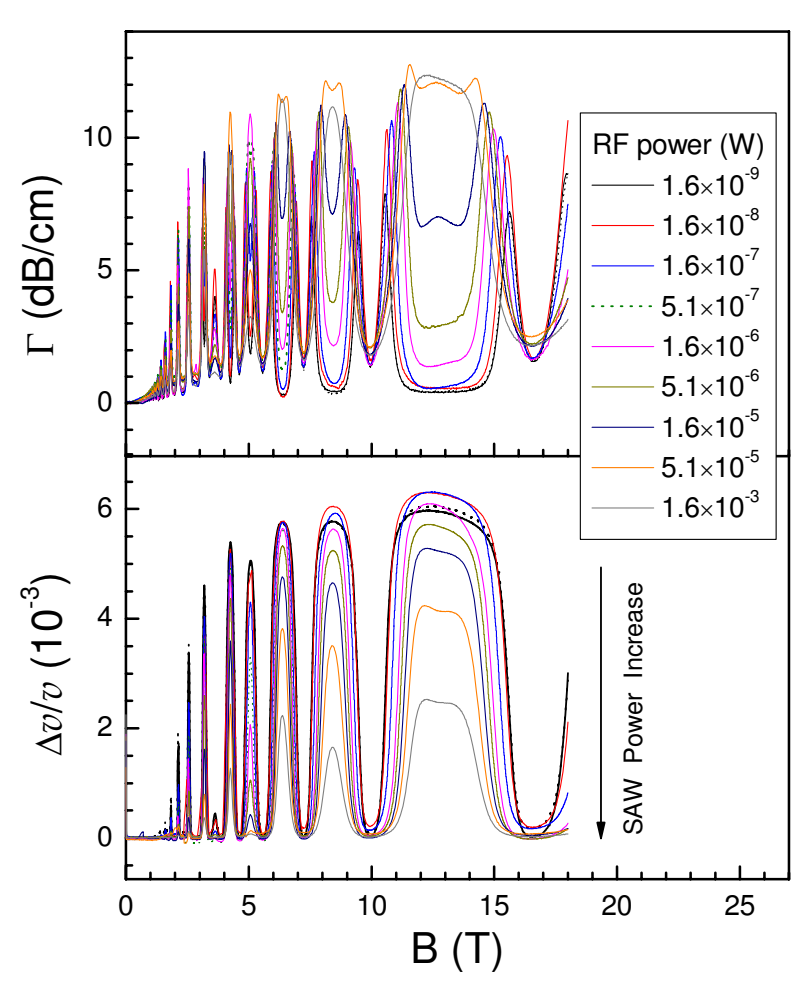

FIG. 8: Dependences of $\Gamma$ and $\Delta v / v(0)$ on the magnetic field at different SAW powers; $f=30 \mathrm{MHz}, T=0.3 \mathrm{~K}$.

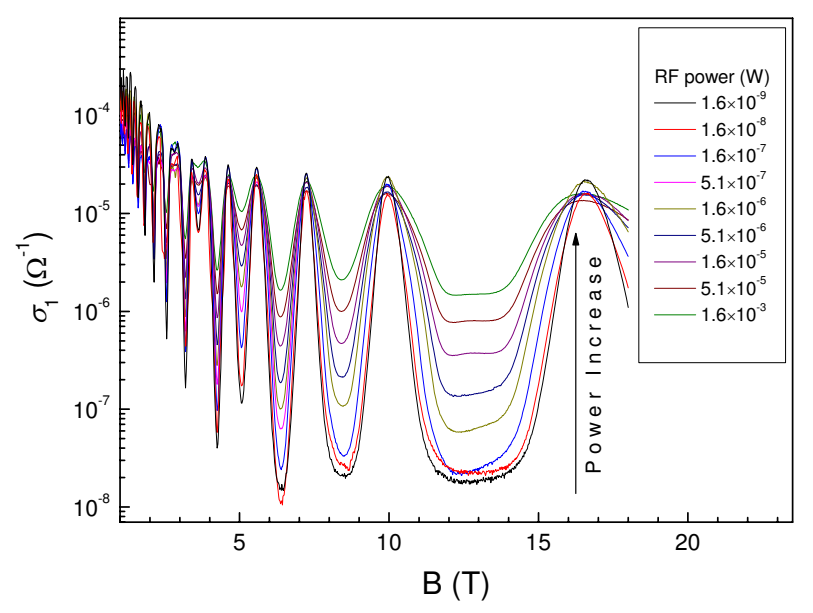

FIG. 9: Dependence of $\sigma_{1}$ on magnetic field at different values of $\mathrm{RF}$ power introduced into the sample; $T=0.3 \mathrm{~K}$.

in the oscillations minima increases while the oscillation amplitudes decrease.

In order to identify the nature of the nonlinear effects we need to know the electric field which accompanies the SAW. According to Ref. 12 this field can be obtained from the following relations:

$$
|E|^{2}=K^{2} \frac{32 \pi}{v}\left(\varepsilon_{1}+\varepsilon_{0}\right) \frac{z q e^{(-2 q(a+d))}}{\left(1+\frac{4 \pi \sigma_{2}(\omega)}{\varepsilon_{s} v} t\right)^{2}+\left(\frac{4 \pi \sigma_{1}(\omega)}{\varepsilon_{s} v} t\right)^{2}} \frac{W}{l},
$$

$$
z=\left[\left(\varepsilon_{1}+\varepsilon_{0}\right)\left(\varepsilon_{s}+\varepsilon_{0}\right)-e^{(-2 q a)}\left(\varepsilon_{1}-\varepsilon_{0}\right)\left(\varepsilon_{s}-\varepsilon_{0}\right)\right]^{-2},
$$

where $W$ is the input SAW power and $l$ is the width of the sound track.

The real part of the ac conductivity $\sigma_{1}$ against the electric field of the surface acoustic wave is plotted in Figure 10 .

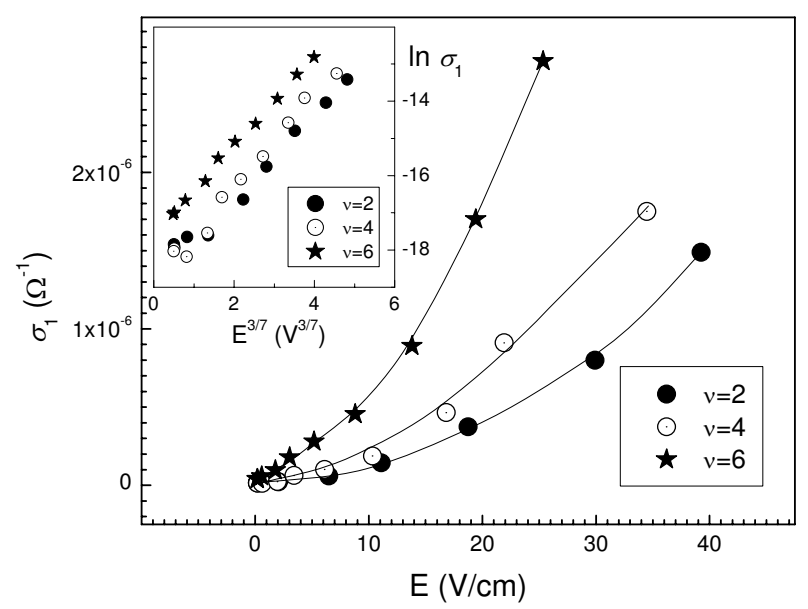

FIG. 10: Real part of the ac conductivity $\sigma_{1}$ vs the SAW electric field for $\nu=2,4,6 ; f=30 \mathrm{MHz}, T=0.3 \mathrm{~K}$. Inset: $\ln \sigma_{1}$ vs $E^{3 / 7}$ for $\nu=2,4,6$. The lines are guides to the eye.

The impact of a strong constant electric field on the electrical conductivity, caused by the activation of charge carriers to the percolation level of the conduction band, was discussed in Ref. 16. The authors argued that a strong electric field reduces the activation energy, which in turn may be interpreted as a lowering of the percolation threshold. For the 2D case the dependence of the conductivity on the electric field is as follows:

$$
\sigma_{1}=\sigma_{1}^{0} \exp \left(\alpha E^{3 / 7} / k_{B} T\right)
$$

with

$$
\alpha=\left(\operatorname{Cel}_{s p} V_{0}\right)^{3 / 7}
$$

where $\sigma_{1}^{0}$ is the conductivity in the linear regime, $C$ is a numerical coefficient, $V_{0}$ is the amplitude of the fluctuations of the random potential, and $l_{s p}$ is the characteristic spatial scale of the potential. In our experiment the conditions $\omega \tau_{p} \ll 1$ was met, where $\omega$ is the SAW frequency and $\tau_{p}$ is the transport relaxation time. Therefore, the wave (SAW) can be considered as stiffened. As a result, one may use the theory obtained for the strong static electric fields to interpret nonlinear effects in the ac conductivity. 16 Although we studied the nonlinear effects at $T=0.3 \mathrm{~K}$, where the conductivity no longer has an activated character but depends weakly on temperature (as expected for the case of "two-site" hopping), the variation of the real conductivity $\sigma_{1}$ with 3 ) the electric field of the surface acoustic wave, as shown 
in Figure 10, is nevertheless well described by the dependence $\ln \sigma_{1} \propto E^{3 / 7}$. One can assume that the nonlinearity mechanism is mixed: heating the electrons in an electric field of the SAW leads to the activated dependence of the conductivity on temperature. The nonlinearity in this case is characterized by nonlinear conduction at the percolation level, as in Ref. 16. Although this statement may require an additional proof it should be noted that a similar behavior was observed in the non-linear hopping conductivity in $\mathrm{n}-\mathrm{GaAs} / \mathrm{AlGaAs}$ heterostructures $\underline{12}$

\section{CONCLUSION}

In this paper we described the results obtained by applying contactless acoustic techniques to the study of the high frequency conductivity in a p-Ge/GeSi heterostructure with very high mobility. The study was carried out at temperatures of 0.3-5.8 $\mathrm{K}$ in the magnetic field of up to $18 \mathrm{~T}$ in both the linear and non-linear regimes. It was shown that in the linear regime at $T<1-2 \mathrm{~K}$ the high frequency conductivity in the IQHE oscillations minima is of a hopping nature and can be described by a "two-site" model. In the nonlinear regime the dependence of the real part of the hopping conductivity on the electric field of the SAW can be accurately described by the functional form $\ln \sigma_{1} \propto E^{3 / 7}$.

By analyzing the temperature dependences of the activated conductivity at odd filling factors, corresponding to the spin-split Landau levels, we were able to determine the g-factor.

\section{Acknowledgments}

This work was supported by the grant of RFBR 11-0200223, a grant of the Presidium of the Russian Academy of Science, the Program "Spintronika" of Branch of Physical Sciences of RAS, grant U.M.N.I.K 16906. A portion of this work was performed at the National High Magnetic Field Laboratory, which is supported by NSF Cooperative Agreement No. DMR-0654118, by the State of Florida, and by the DOE. We thank E. Palm, T. Murphy, J.H. Park, and G. Jones for technical assistance during the experiments.
1 I.L. Drichko, A.M. Diakonov, E.V. Lebedeva, I.Yu. Smirnov, O.A. Mironov, M. Kummer, and H. von Känel, J. Appl. Phys. 106, 094305 (2009).

2 C. Rosenblad, H.R. Deller, A. Dommann, T. Meyer, P. Schroeter, and H. von Känel, J. Vac. Sci. Technol. A 16, 2785 (1998).

3 H. von Känel, M. Kummer, G. Isella, E. Müller, T. Hackbarth, Appl. Phys. Lett. 80, 2922 (2002).

4 O.A. Kuznetsov, A.L. Chernov, L.K. Orlov, R.A. Rubtsova, Yu.G. Arapov, N.A. Gorodilov, G.L. Shtrapenin, JETP Letters 54, 347 (1991); Pis'ma Zh. Eksp. Teor. Fiz. 54, 351 (1991).

5 Yu.G. Arapov, N.A. Gorodilov, O.A. Kuznetsov, V.N. Neverov, L.K. Orlov, R.A. Rubtsova, G.I. Kharus, A.L. Chernov, N.G. Shelushinina, G.L. Shtrapenin, Semiconductors 27, 642 (1993); Fiz. Tekh. Poluprovodn. 27, 1165 (1993).

6 N.A. Gorodilov, O.A. Kuznetsov, L.K. Orlov, R.A. Rubtsova, A.L. Chernov, N.G. Shelushinina, G.L. Shtrapenin, JETP Letters 56, 394 (1992); Pis'ma Zh. Eksp. Teor. Fiz. 56, 409 (1992).

7 Yu.G. Arapov, N.A. Gorodilov, V.N. Neverov, G.I. Kharus, N.G. Shelushinina, O.A. Kuznetsov, L.K. Orlov, R.A. Rubtsova, A.L. Chernov, JETP Letters 59, 245 (1994); Pis'ma Zh. Eksp. Teor. Fiz. 59, 227 (1994).

8 M.I. D'yakonov, A.V. Khaetskii, JETP, 55917 (1982); Zh.
Eksp. Teor. Fiz. 82, 1584 (1982).

9 T. Irisawa, M.Myronov, O.A. Mironov, E.H.C. Parker, K. Nakagawa, M. Murata, S. Koh, Y. Shiraki, Appl. Phys. Lett. 82, 1425 (2003).

10 B. Rössner, B. Batlogg, H. von Känel, D. Chrastina, G. Isella, Mat. Sc. Semicond. Proc. 9, 777 (2006).

11 A. Wixforth, J. Scriba, M. Wassermeier, J. P. Kotthaus, G. Wiemann, and Schlapp, Phys. Rev. B 40, 7874 (1989); A. Wixforth, J.P. Kotthaus, and G. Weimann, Phys. Rev. Lett. 56, 2104 (1986).

12 I.L. Drichko, I.Yu. Smirnov, A.V.Suslov, D.R. Leadley, Phys. Rev. B 83, 235318 (2011).

13 M. Pollak and T. Geballe, Phys. Rev. 122, 1742 (1961); Yu.M. Galperin, V.L. Gurevich, and D.A. Parshin, in Hopping Transport in Solid, edited by B. Shklovskii and M. Pollak (Elsevier, New York 1991).

14 A.L. Efros, JETP 89, 1057 (1985); Zh. Eksp. Teor. Fiz. 89, 1834 (1985).

15 Yu.G. Arapov, G.I. Harus, N.G. Shelushinina, M.V. Yakunin, V.N. Neverov, O.A. Kuznetsov, L. Ponomarenko and A. De Visser, Low Temp. Phys. 30, 867 (2004); Fiz. Nizk. Temp. 30, 1157 (2004).

16 B.I. Shklovskii, Sov. Phys. Semicond. 13, 53 (1979); Fiz. Tekh. Poluprovodn. (Leningrad) 13, 93 (1979). 